\title{
Effect of nanoparticles on the thermal behaviour of a NePCM- based heat sink
}

\author{
Hamza Faraji ${ }^{1, *}$, Mustapha Faraji ${ }^{1}$, and Mustapha El Alami ${ }^{1}$ \\ ${ }^{1}$ Physics Department, LPMMAT Laboratory, Faculty of Sciences Ain Chock, Hassan II University, Casablanca- Morocco
}

\begin{abstract}
This work presents a computational investigation of the passive cooling of an electronic component in a latent heat storage unit filled with nano-enhanced phase change material (NePCM). The electronic component is flush mounted on a substrate (motherboard) in the centre of the bottom wall of a rectangular enclosure. This electronic component generates heat at a constant and uniform volumetric rate. A 2D mathematical model based on the conservation equations of mass, momentum and energy has been developed using the enthalpy- porosity method. The effect of natural convection in the molten NePCM is considered during the melting process. The centrepiece of this study is to improve the functionality of PCM trough insertion of nanoparticles. Computational surveys have been developed to evaluate the effect of volumetric concentration as well as the nanoparticles type by monitoring the evolution of the maximum temperature of the electronic component, the average Nusselt number and the velocity field. These investigations show that the volumetric concentration and the nanoparticles type are two main factors to take into account for an improvement in performance of the NePCM-based heat sink.
\end{abstract}

\section{Introduction}

The last years of the $20^{\text {th }}$ century were decisive for the energy state of the countries after the oil crisis in 1973. The latter and the increase in energy prices prompted several researchers to trigger research studies focusing on replacing traditional energy resources with new and more efficient ones. Storage remains a recommended way for optimal management of thermal energy. It makes it possible to adapt the production to the needs. Heat can be stored in three ways [1]: sensible storage, latent storage and thermochemical storage. Latent storage has been of great interest to researchers because of the important role played during the melting process for storing heat. PCMs are candidates that can satisfy different criteria necessary for latent storage from the first appearance on aeronautical fields [2,3]. These materials are characterized by their high storage density given them application in different domains; the cooling of electronic components, the efficiency of building, agricultural greenhouses ... [4,6].

The unacceptable disadvantage of PCMs is their low thermal conductivity. This feature weakens the heat transfer within enclosures filled with PCM. Various technics are announced to improve the thermal conductivity of PCMs and thereafter heat transfer. The insertion of metallic nanoparticles has been of great interest to researchers. Ebrahimi and Dadvand [7] have numerically studied the melting of NePCM with alumina $\mathrm{Al}_{2} \mathrm{O}_{3}$ nanoparticles. This melting process takes place in a rectangular enclosure containing two isothermal heat sources. Two are exposed to a cold temperature and the rest are hot. The impact of the nanoparticle load is analysed and the results showed that in all the studied cases, a concentration of $2 \%$ in alumina nanoparticles would give the highest melting rate and the best heat transfer within the enclosure. In spherical enclosures, Hosseinizadeh et al. [8] investigated numerically the melting of a PCM (RT27) enhanced by copper nanoparticles. It is inferred that a suspension of copper nanoparticles increases the effective thermal conductivity due to a decrease in latent heat that will in turn increase the melting rate. Experimental studies are also carried out in this axe. Ho and Gao [9] have experimentally processed the n-octadecane PCM melting process dispersed by Alumina metal nanoparticles. The results showed that heat transfer governed by natural convection in liquid PCM weakens with increasing concentration of dispersed nanoparticles compared to the case of the base PCM.

The aim of this study is to investigate numerically the effect of nanoparticle insertion in a rectangular enclosure filled with PCM and heated from below by an electronic component. It is clear that all the studies, quoted above, treat the heat transfer with isothermal heat sources. The specificity of this study lies in the fact that the heating is achieved by a constant volumetric generation of heat and the electronic component is mounted on a conductive wall acting as a fin. The hydrodynamic behaviour of different nanoparticles type is examined.

\footnotetext{
* Corresponding author: faraji-ham@hotmail.com
} 


\section{Analysis and modelling}

The concern of this study is to investigate numerically the melting of a NePCM, for different types of nanoparticles, in a rectangular enclosure with an aspect ratio of $\mathrm{A}=0.5$. The enclosure contains a protruding electronic component flush-mounted on a substrate in the centre of its bottom wall. A constant volumetric heat generation is taken into account. The substrate plays the role of a motherboard that can be considered as a conductive wall. All walls of the enclosure are adiabatic. The physical model describing this configuration is given in Figure 1. The insertion of nanoparticles is introduced for reasons of increasing the thermal conductivity of PCM.

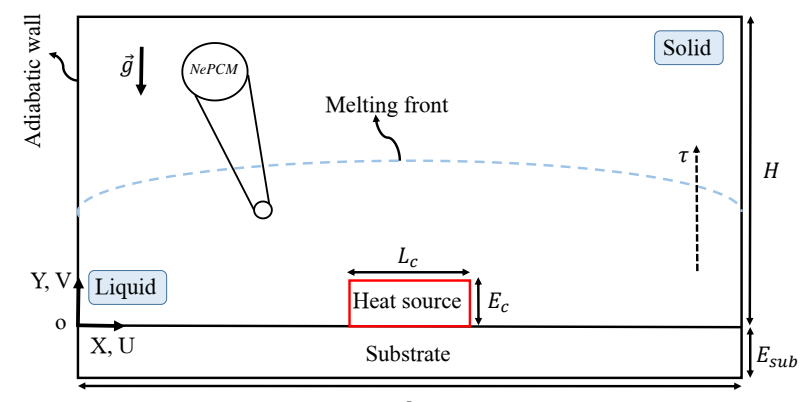

Fig. 1. The physical model.

The non-dimensional governing general equation of the studied physical problem, taking into account the buoyancy force with terms grouped in Table 1, can be expressed as follows:

$$
\frac{\partial \phi}{\partial \tau}+\frac{\partial(U \phi)}{\partial X}+\frac{\partial(V \phi)}{\partial Y}=\Gamma \frac{\partial^{2} \phi}{\partial X^{2}}+\Gamma \frac{\partial^{2} \phi}{\partial Y^{2}}+S_{\phi}
$$

Table 1. Terms of general equation.

\begin{tabular}{|l|c|c|c|}
\hline Equation & $\phi$ & $\Gamma$ & $S_{\phi}$ \\
\hline Continuity equation & 1 & 0 & 0 \\
\hline Energy equation & $\theta$ & $\bar{\alpha}$ & $S_{\theta}$ \\
\hline $\begin{array}{l}\text { Conservation equation of } \\
\text { momentum U }\end{array}$ & $U$ & $P r$ & $-\frac{\partial P}{\partial X}+S_{U}$ \\
\hline $\begin{array}{l}\text { Conservation equation of } \\
\text { momentum V }\end{array}$ & $V$ & $P r$ & $-\frac{\partial P}{\partial Y}+S_{V}$ \\
\hline
\end{tabular}

With:

$$
\begin{gathered}
S_{U}=-\bar{C} \frac{(1-f)^{2}}{b+f^{3}} U+R a \operatorname{Pr} \cos (\Omega) \\
S_{V}=-\bar{C} \frac{(1-f)^{2}}{b+f^{3}} V+R a \operatorname{Pr} \sin (\Omega) \\
S_{\theta}=\delta_{1}\left(\left(\delta_{2}-1\right) \frac{1}{\text { Ste }} \frac{\partial f}{\partial \tau}+\frac{Q \delta_{2}}{E_{c} L_{c}}\right)
\end{gathered}
$$

The non-dimensional governing variables of the problem are grouped below:

$$
\begin{gathered}
X=\frac{x}{l_{0}}, Y=\frac{y}{l_{0}}, \tau=\frac{\alpha_{m, l} t}{l_{0}^{2}} \\
U=\frac{l_{0} u}{\alpha_{m, l}}, \mathrm{~V}=\frac{l_{0} v}{\alpha_{m, l}}, \theta=\frac{\left(T-T_{m}\right) k_{m, l}}{Q} \\
E_{c}=\frac{e_{c}}{l_{0}}, \mathrm{~L}_{c}=\frac{l_{c}}{l_{0}}, \mathrm{~A}=\frac{H}{L} \quad, \quad \mathrm{E}_{s u b}=\frac{e_{s u b}}{l_{0}}
\end{gathered}
$$

All the enclosure walls are adiabatic:

$$
\left.\frac{\partial \theta}{\partial \eta}\right|_{\text {wall }}=0 \quad, \quad \eta \perp \text { wall }
$$

For the interface between two different materials (1 and 2); substrate, PCM and electronic component:

$$
-\left.K_{1} \frac{\partial \theta}{\partial \eta}\right|_{\text {interface }}=-\left.K_{2} \frac{\partial \theta}{\partial \eta}\right|_{\text {interface }} \quad, \quad \theta_{1}=\theta_{2}
$$

Initially, the following conditions are satisfied:

$$
\theta=0 \quad, \quad \mathrm{U}=V=0 \quad, \quad f=0
$$

The thermo-physical properties of NePCM are calculated using the following expressions:

$$
\begin{gathered}
(\rho L)_{N e P C M}=\left(1-X_{n}\right)(\rho L)_{P C M} \\
\left(\rho c_{p}\right)_{N e P C M}=X_{n}\left(\rho c_{p}\right)_{P}+\left(1-X_{n}\right)\left(\rho c_{p}\right)_{P C M}
\end{gathered}
$$

The effective thermal conductivity is calculated based on the combination of Maxwell's theory [10] (left term) and Brownian motion [11] (term on the right):

$$
K_{\text {eff }} k_{m}=\frac{k_{p}+2 k_{m}-2 X_{n}\left(k_{m}-k_{p}\right)}{k_{p}+2 k_{m}+X_{n}\left(k_{m}-k_{p}\right)} k_{m}+K_{\text {Brown }}
$$

With :

$$
\begin{gathered}
K_{\text {Brown }}=510^{4} \beta \xi X_{n} \rho_{m} c_{p, m} \sqrt{\frac{B T}{\rho_{p} d_{p}}} f\left(T, X_{n}\right) \\
\beta=9.881\left(100 X_{n}\right)^{-0.9446}
\end{gathered}
$$

and the function $f\left(T, X_{n}\right)$ is derived from experimental data[11].

\section{Validation}

A validation of the predictions of our numerical model was realized by repetition of the experimental tests of Zhang et al. [12]. The studied configuration represents a rectangular enclosure filled with n-octadecane and heated discretely by three heat sources flush-mounted on 
its left vertical wall. The remaining walls are adiabatic. The dimensions of the enclosure are $100 \mathrm{~mm} \times 60 \mathrm{~mm}$. Figure 2 shows a comparison between the results of our simulations and those obtained by Zhang et al. [12] in terms of the position of the melting front. Given the complexity of the phenomenon, an analysis of such figure reveals a sufficient agreement between the present and the published experimental results of Zhang et al. [12]. A difference is clearly observed at the upper part by advancement of the experimental melting front on the numerical one obtained by our simulations. Indeed, this difference is due to an expansion of a quantity of liquid n-octadecane during the melting process. Another factor contributing to the discrepancy between numerical predictions and experimental observations is the difficulty throughout the experiment in maintaining a constant and uniform power of the three heat sources.

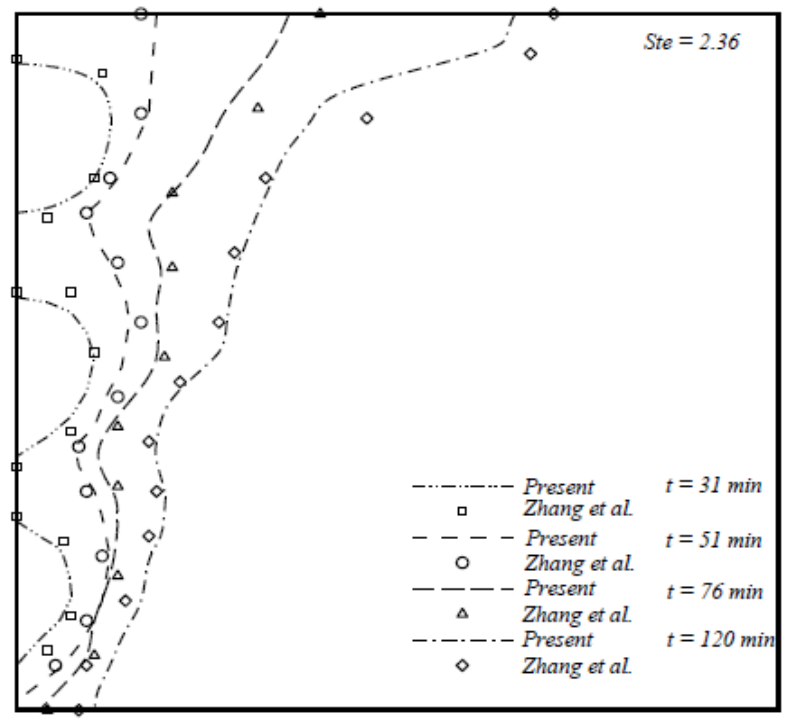

Fig. 2. Numerical and experimental melting front positions.

\section{Results and discussion}

In the present work, a numerical investigation of the melting of a PCM (n-eicosane) dispersed with different types of metallic nanoparticles $\left(\mathrm{Al}_{2} \mathrm{O}_{3}, \mathrm{ZnO}, \mathrm{CuO}\right.$ and $\mathrm{Cu}$ ) in a rectangular enclosure of an aspect ratio $\mathrm{A}=0.5$. The thermo-physical properties of the PCM, electronic component, substrate and metallic nanoparticles used in this work are given in Tables 2 and 3.

\subsection{Liquid fraction (a measure of the melting rate)}

Instead of an explicit monitoring of the solid liquid interface, a calculation of the liquid fraction that measures the melting rate is made for four types of nanoparticles at a volumetric concentration of $2 \%$. Figure 3 shows the time evolution of the liquid fraction. The first clear remark from Figure 3 is a similar, linear and rapid initial evolution for the four types of nanoparticles.
Table 2. Thermo-physical properties of materials [13, 14].

\begin{tabular}{|l|l|l|}
\hline \multicolumn{1}{|c|}{ Electronic device } & \multicolumn{1}{|c|}{ PCM } & \multicolumn{1}{c|}{ Substrate } \\
\hline$k_{c}=170 \mathrm{~W} / \mathrm{m} . \mathrm{K}$ & $k_{m}=0.1505 \mathrm{~W} / \mathrm{m} \cdot \mathrm{K}$ & $k_{s}=19.7 \mathrm{~W} / \mathrm{m} \cdot \mathrm{K}$ \\
$\rho_{c}=3260 \mathrm{~kg} / \mathrm{m}^{3}$ & $\rho_{m}=769 \mathrm{~kg} / \mathrm{m}^{3}$ & $\rho_{s}=3900 \mathrm{~kg} / \mathrm{m}^{3}$ \\
$c_{P}=740 \mathrm{~J} / \mathrm{kg}$ & $c_{P}=2460 \mathrm{~J} / \mathrm{kg}$ & $c_{P}=900 \mathrm{~J} / \mathrm{kg}$ \\
$T_{c r}=98^{\circ} \mathrm{C}$ & $\mu=4.1510^{-3} \mathrm{~m}^{2} / \mathrm{s}$ & \\
& $\beta=8.0510^{-4} 1 / \mathrm{K}$ & \\
& $\Delta H=2.4710^{5} \mathrm{~J} / \mathrm{kg}$ & \\
& $T_{m}=36^{\circ} \mathrm{C}$ & \\
\hline
\end{tabular}

Table 3. Thermo-physical properties of nanoparticles [13, 14].

\begin{tabular}{|c|c|c|c|c|}
\hline & $\boldsymbol{A l}_{2} \boldsymbol{O}_{3}$ & $\boldsymbol{Z n O}$ & $\boldsymbol{C u} \boldsymbol{O}$ & $\boldsymbol{C u}$ \\
\hline$\rho_{P}\left(\mathrm{~kg} / \mathrm{m}^{3}\right)$ & 3600 & 5606 & 6510 & 8954 \\
\hline$\left(c_{p}\right)_{P}(J / k g)$ & 765 & 514 & 540 & 383 \\
\hline$k_{P}(\mathrm{~W} / \mathrm{m} \cdot \mathrm{K})$ & 36 & 23.4 & 18 & 400 \\
\hline
\end{tabular}

Indeed, initially the heat transfer in the vicinity of the heat source is, practically, purely conductive. This is clearly proven in the evolution of the maximum temperature of the heat source and the average Nusselt number. The effect of the type of nanoparticles is also clearly shown. For the same concentration, the higher melting rate corresponds to an insertion of copper $\mathrm{Cu}$ nanoparticles while the low melting rate is noted for the case of alumina $\mathrm{Al}_{2} \mathrm{O}_{3}$ nanoparticles dispersion.

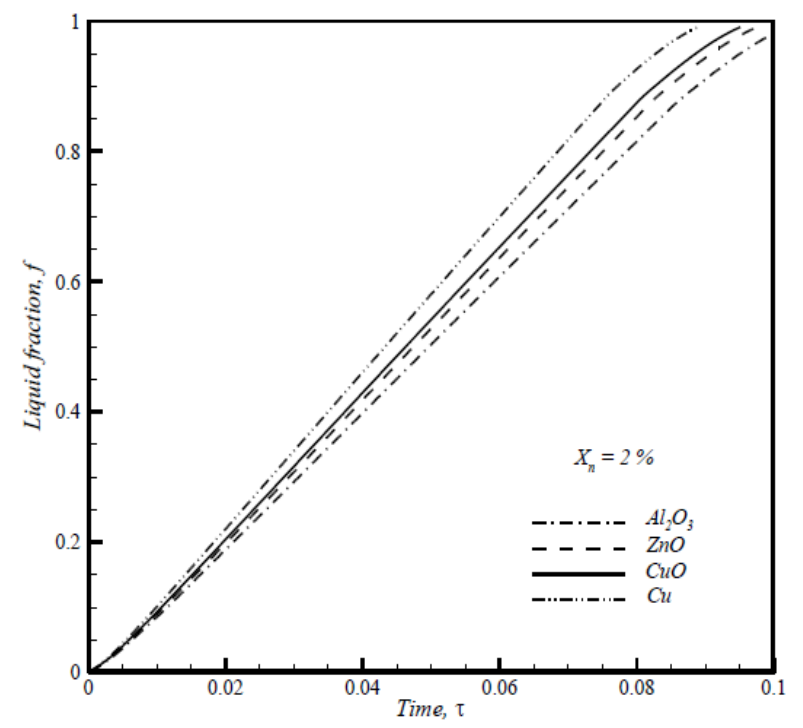

Fig. 3. Liquid fraction variation for different types of nanoparticles.

\subsection{Maximal operating temperature}

The evolution of the maximum operating temperature of the heat source is shown in Figure 4. This evolution is divided into three stages. The first stage consists of a rapid and linear evolution of the temperature spread over the same time interval where the liquid fraction also evolves in the same way. The effect of the nanoparticle type is negligible along this stage because the heat transfer is purely conductive. In fact, all the heat produced by the heat source is stored sensibly within it 
and the substrate while causing a rapid increase in the operating temperature. This behavior is also visualized in the evolution of the average Nusselt number shown in Figure 5. The second stage is characterized by a constant operating temperature "plateau". In this stage, natural convection clearly contributes to the cooling of the heat source while keeping a constant operating temperature. The heat transfer, during this stage, is constant as well as no sensible storage takes place, see Figure 5. It is important to note that between this second stage and the first one there will be a competition between cooling and heating of the heat source given the flow of cold and hot currents within the enclosure. This competition is illustrated by the appearance of fluctuations in Figure 5. A third stage is also shown with a further increase in the operating temperature of the heat source. In this stage, conduction and natural convection are present. This evolution in three stages is repeated in the same way concerning that of the average Nusselt number, see Figure 5.

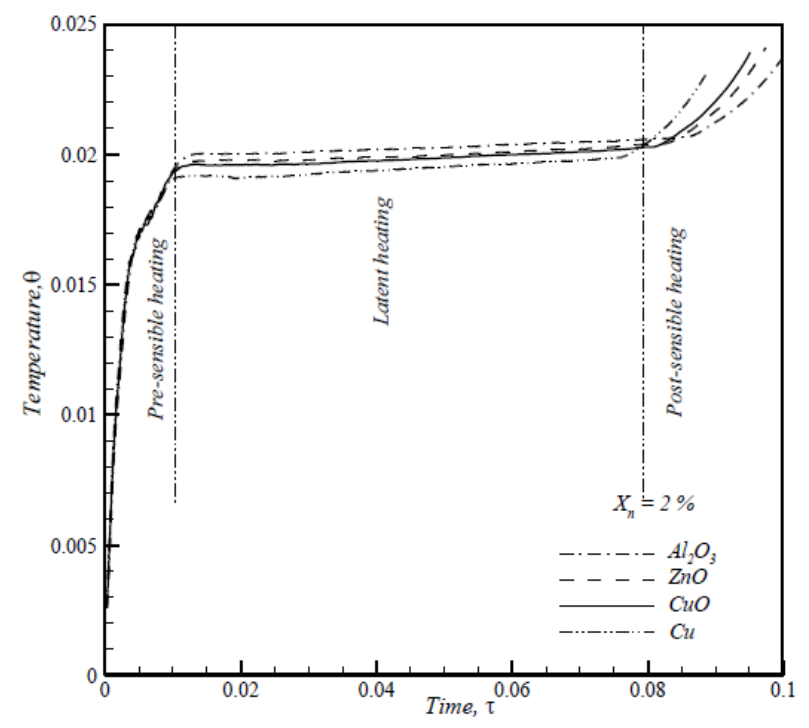

Fig. 4. Maximal operating temperature evolution for different types of nanoparticles.

The insertion of copper $\mathrm{Cu}$ nanoparticles guarantees a minimum operating temperature compared to other types followed by that of Copper Oxide $\mathrm{CuO}$. By inserting copper nanoparticles, the heat source operates at a minimum temperature but during a minimum secure operating time (the plateau duration) compared to that visualized for other types of nanoparticles. Hence the choice of working for a longer period in a safe and at maximum temperature or working for a shorter period and with a minimum temperature.

\subsection{Isotherms and streamlines evolution}

Figure 6 shows the evolution of isotherms (top) and streamlines (down) at $\tau=0.04$ for the four types of nanoparticles. It is clear that during the melting process, a temperature difference between the upper and lower region of the liquid NePCM will occur. This difference will cause a difference in density and subsequently a descent of the cold liquid and a rise of the hot one. This gives rise to two convective cells; one in the clockwise direction and the other in the opposite direction. It is clear that in the region of intersection of the two convective cells, there is a plume structure (wall jet). This structure reflects the contribution of natural convection to the cooling of the heat source. It is important to note that the minimum temperature of the heat source is noted for the case of insertion of $\mathrm{Cu}$ nanoparticles, as well as in this case the flow intensity $\Psi_{\max }$ is higher. It is clear that more than the density of the nanoparticles increases more than the maximum operating temperature of the heat source decreases and the flow intensity increases. Hence, a maximum thermal performance is proved in the case of insertion of highdensity nanoparticles. Indeed, more than the density increases more than the effect of buoyancy is greater and subsequently an intense flow of liquid NePCM while resulting in a good cooling of the heat source.

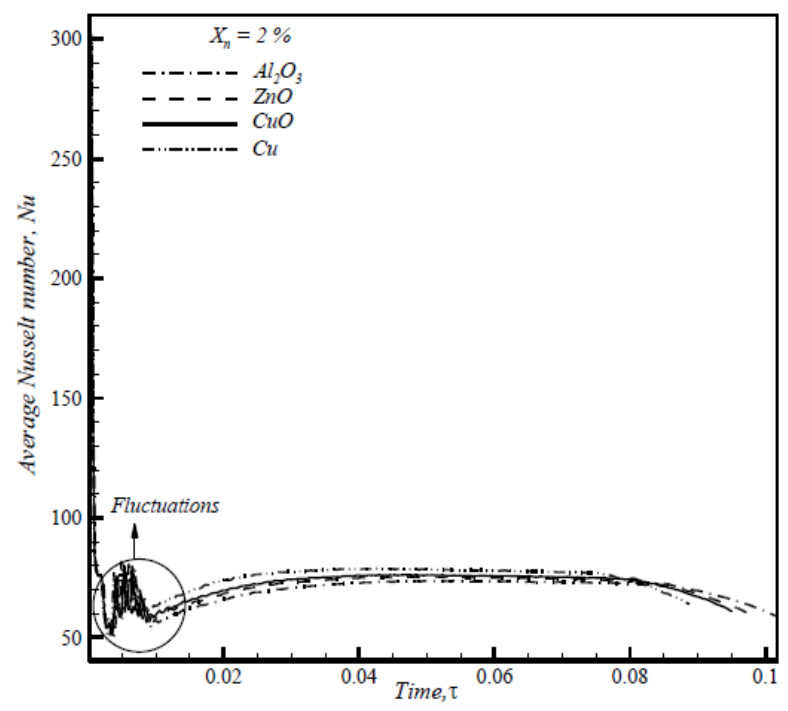

Fig. 5. Average Nusselt number evolution for different types of nanoparticles.

\section{Conclusion}

A numerical investigation of the melting of a NePCM in the presence of four types of nanoparticles in a rectangular enclosure containing a protruding heat source has been performed. The effect of nanoparticles types on the thermal performance of the heat sink is analyzed. It is found that the insertion of higher density and thermal conductivity nanoparticles increases the melting rate and improves the thermal response of the heat sink to the cooling of the heat source. 

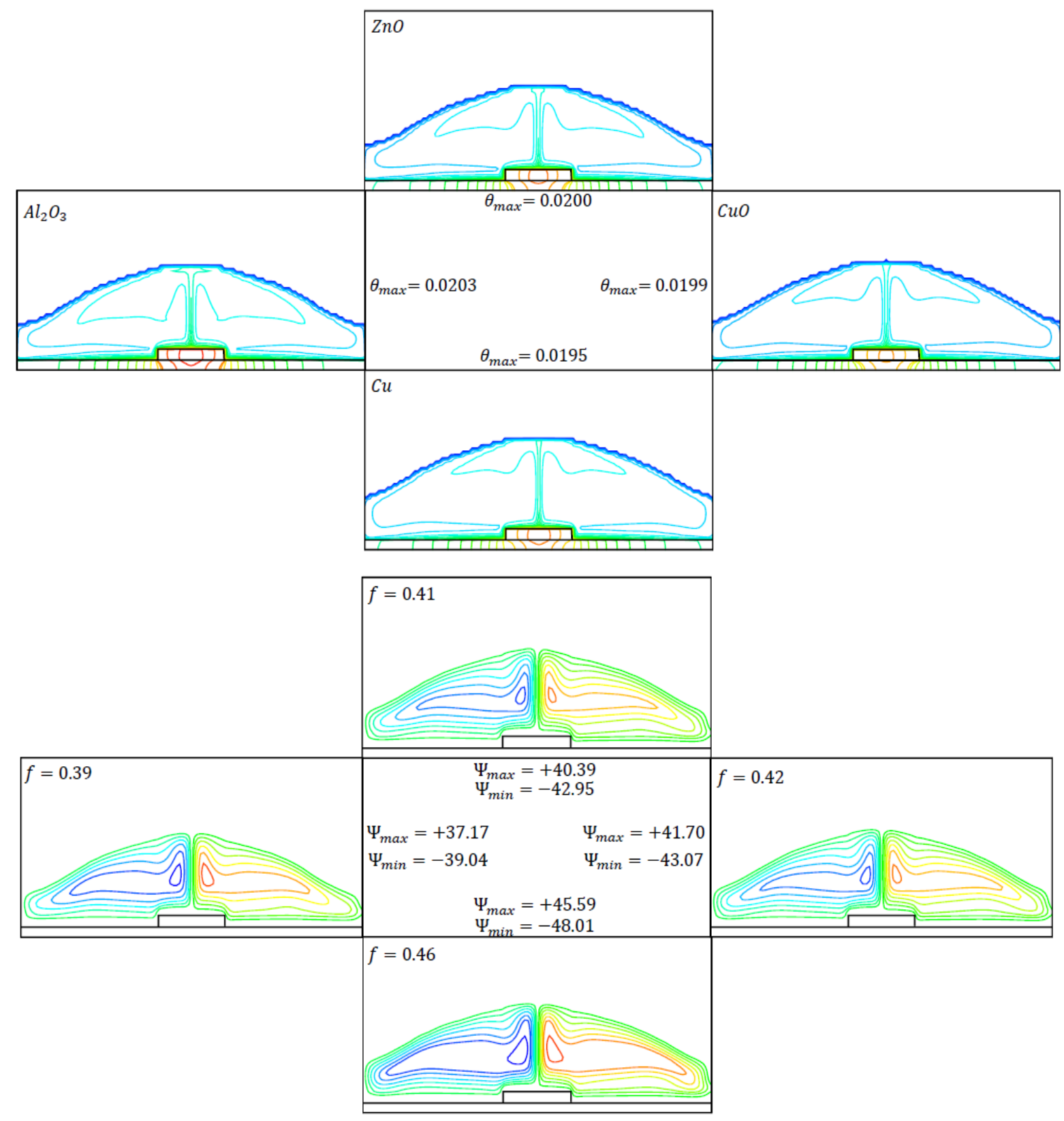

Fig. 6. Isotherms (top) and streamlines (down) for different types of nanoparticles at $\tau=0.04$.

\section{References}

[1] K. Pielichowska, K. Pielichowski, Progress in Materials Science, 65, 67 (2014).

[2] A. Abhat, Solar Energy, 30-4, 313 (1983).

[3] W. Humphries, E. Griggs, NY, USA: NASA Technical Paper (1977).

[4] M. Faraji, Journal of microelectronics and electronic packaging, 7-2, 79 (2010).

[5] B. Zalba, J. M. Marin, L. F. Cabeza, H. Mehling, Applied Thermal Engineering, 23, 251 (2003).

[6] F. Berroug, E. K. Lakhal, M. El Omari, M. Faraji, H. El Qarnia, Journal of Thermal Science, 20-4, 377 (2011).

[7] A. Ebrahimi, A. Dadvand, Alexandria Engineering Journal, 54, 1003 (2015).
[8] S.F. Hosseinizadeh, A.A. R. Darzi, F.L. Tan, International Journal of Thermal Sciences, 51, 77 (2012).

[9] C.J. Ho, J.Y. Gao, International Journal of Heat and Mass Transfer, 62, 2 (2013).

[10] J. A. Maxwell, Oxford University Press, Cambridge, UK (1904).

[11] R. S. Vajjha, D. K. Das, International Journal of Heat and Mass Transfer, 52, 4675 (2009).

[12] Y. Zhang, Z. Chen, Q. Wang and Q. Wu, Experimental Thermal Fluid Science, 6, 196 (1993).

[13] W. Humphries, E. Griggs, NY, USA: NASA Technical Paper (1977).

[14] R. R. Tummala, $1^{\text {st }}$ Ed. McGraw-Hill Education (2001). 